\title{
The LHCf experiment: recent physics results
}

\author{
E. Berti ${ }^{*}{ }^{a b}$ O. Adriani, ${ }^{a b}$ L. Bonechi, ${ }^{a}$ M. Bongi, ${ }^{a b}$ R. D'Alessandro, ${ }^{a b}$ \\ M. Haguenauer, ${ }^{c}$ Y. Itow, ${ }^{d e}$ K. Kasahara, ${ }^{f}$ Y. Makino, ${ }^{d}$ K. Masuda, ${ }^{d}$ H. Menjo, ${ }^{g}$ \\ Y. Muraki, ${ }^{d}$ K. Ohashi, ${ }^{d}$ P. Papini, ${ }^{a}$ S. Ricciarini, ${ }^{a h}$ T. Sako,${ }^{i}$ N. Sakurai, ${ }^{j}$ K. Sato, ${ }^{d}$ \\ M. Shinoda, ${ }^{d}$ T. Suzuki, ${ }^{f}$ T. Tamura, ${ }^{k}$ A. Tiberio, ${ }^{a b}$ S. Torii, ${ }^{f}$ A. Tricomi, ${ }^{l m}$ \\ W. C. Turner, ${ }^{n}$, M. Ueno, ${ }^{d}$ Q. D. Zhou ${ }^{d}$
}

${ }^{a}$ INFN Section of Florence - Florence, Italy

${ }^{b}$ University of Florence - Florence, Italy

${ }^{c}$ Ecole-Polytechnique - Palaiseau, France

${ }^{d}$ Institute for Space-Earth Environmental Research - Furo-cho, Chikusa-ku, Nagoya, Japan

${ }^{e}$ Kobayashi-Maskawa Institute for the Origin of Particles and the Universe, Nagoya University -

Nagoya, Japan

${ }^{f}$ RISE, Waseda University - Shinjuku, Tokyo, Japan

${ }^{g}$ Graduate School of Science, Nagoya University - Nagoya, Japan

${ }^{h}$ IFAC-CNR - Florence, Italy

${ }^{i}$ Institute for Cosmic Ray Research, University of Tokyo, Chiba, Japan

${ }^{j}$ Tokushima University, Tokushima, Japan

${ }^{k}$ Kanagawa University - Kanagawa, Japan

${ }^{l}$ INFN Section of Catania - Catania, Italy

${ }^{m}$ University of Catania - Catania, Italy

${ }^{n}$ LBNL - Berkeley, California, USA

E-mail: eugenio.berti@fi.infn.it

The main aim of the LHCf experiment is to provide precise measurements of the production spectra relative to neutral particles produced in the very forward region by high energy protonproton and proton-ion collisions. This information is necessary in order to test and tune hadronic interaction models used by ground-based cosmic rays experiments. In order to reach this goal, LHCf makes use of two small sampling calorimeters installed in the LHC tunnel at $\pm 140 \mathrm{~m}$ from IP1, able to detect neutral particles having pseudo-rapidity $\eta>8$.4. After the operations relative to p-p collisions at $\sqrt{s}=13 \mathrm{TeV}$, the collaboration focused the activity on the study of forward photons and neutrons production at this energy. In this paper, we discuss the recent results relative to the photons analysis, regarding, in particular, the inclusive production cross section, the role of diffractive and non-diffractive processes in forward photons production (investigated thanks to the LHCf-ATLAS joint analysis) and the forward differential energy flow.

PACS: $13.85 .-t, 13.85 . \mathrm{Tp}$

XXXIX International Conference on High Energy Physics

4-11 July 2018

Seoul, Korea

${ }^{*}$ Speaker. 


\section{Introduction}

In order to understand the mechanisms responsible for acceleration and propagation of cosmic rays in the universe, measurements of their flux and composition up to the Greisen-ZatsepinKuzmin (GZK) cut-off are necessary. These measurements are performed by ground-based experiments through the indirect detection of the extensive air showers (EASs) that cosmic rays form when interacting with the atmosphere. The properties of the primary particle are then reconstructed making use of Monte Carlo simulations that, being EASs physics described by soft QCD, necessarily rely on phenomenological models. The deviation among them is very significant at high energies, resulting in large systematic uncertainties on cosmic rays measurements. The main aim of the LHC forward (LHCf [1]) experiment is to give important information for the calibration of hadronic interaction models in the phase space relevant for EASs physics. This is accomplished measuring the distribution of neutral particles produced in the very forward region by high energy proton-proton and proton-ion collisions. The Large Hadron Collider (LHC [2]) at CERN is the most suitable place where to perform these measurements, because a center of mass energy of $\sqrt{s}=13$ $\mathrm{TeV}$ in p-p collisions is equivalent to about $9 \times 10^{16} \mathrm{eV}$ in the reference frame where the target is at rest, an energy not so distant from the one of Ultra High Energy Cosmic Rays (UHECRs).

\section{The experiment}

The LHCf experiment consists of two small sampling calorimeters installed in the LHC tunnel at $\pm 140 \mathrm{~m}$ from IP1, the interaction point dedicated to ATLAS [3]. Being placed after the D1 dipole magnet, only neutral particles produced in $\mathrm{p}-\mathrm{p}$ and $\mathrm{p}$-ion collisions and having pseudorapidity $\eta>8.4$ can reach the experiment. Each one of the two detectors, called Arm1 and Arm2, is made up by two square towers of $22 \mathrm{~W}$ and $16 \mathrm{GSO}$ layers for a total length of $29 \mathrm{~cm}$, equivalent to $44 X_{0}$ and $1.6 \lambda_{I}$. Towers size is $20 \mathrm{~mm} \times 20 \mathrm{~mm}$ and $40 \mathrm{~mm} \times 40 \mathrm{~mm}$ for Arm1, $25 \mathrm{~mm}$ $\times 25 \mathrm{~mm}$ and $32 \mathrm{~mm} \times 32 \mathrm{~mm}$ for Arm2. Energy resolution is better than 5\% for $\gamma_{\mathrm{s}}$ above 100 $\mathrm{GeV}$ and about $40 \%$ for hadrons above $500 \mathrm{GeV}$. The transverse position of the incident particle is reconstructed using 4 xy imaging layers inserted at different depths. They are formed by $1 \mathrm{~mm}$ width GSO-bars in the case of Arm1 and by $160 \mu \mathrm{m}$ read-out pitch silicon microstrip detectors in the case of Arm2. Position resolution is better than $200 \mu \mathrm{m}$ for $\gamma_{\mathrm{s}}$ above $100 \mathrm{GeV}$ and $1 \mathrm{~mm}$ for hadrons above $500 \mathrm{GeV}$. More detailed descriptions of the detector are reported elsewhere $[4,5]$.

\section{Analysis results}

The data relative to $\mathrm{p}-\mathrm{p}$ collisions at $\sqrt{s}=13 \mathrm{TeV}$ was acquired by the LHCf experiment during Fill 3855, a low luminosity and high $\beta^{*}$ fill. Two main analyses have been carried on so far on this data set, relative, respectively, to photons and neutrons production in the forward region. While the latter analysis has just been submitted for publication, the former analysis has already been published [6]. Because of this reason, here we discuss only the results relative to photons, that were object of more detailed studies by the collaboration. Indeed, thanks to the fact that the LHCf and ATLAS experiments had common operations during data taking, it was possible not only to measure the inclusive photons production using LHCf alone, but also to separate diffractive and non-diffractive production mechanisms using an event tag in ATLAS. 

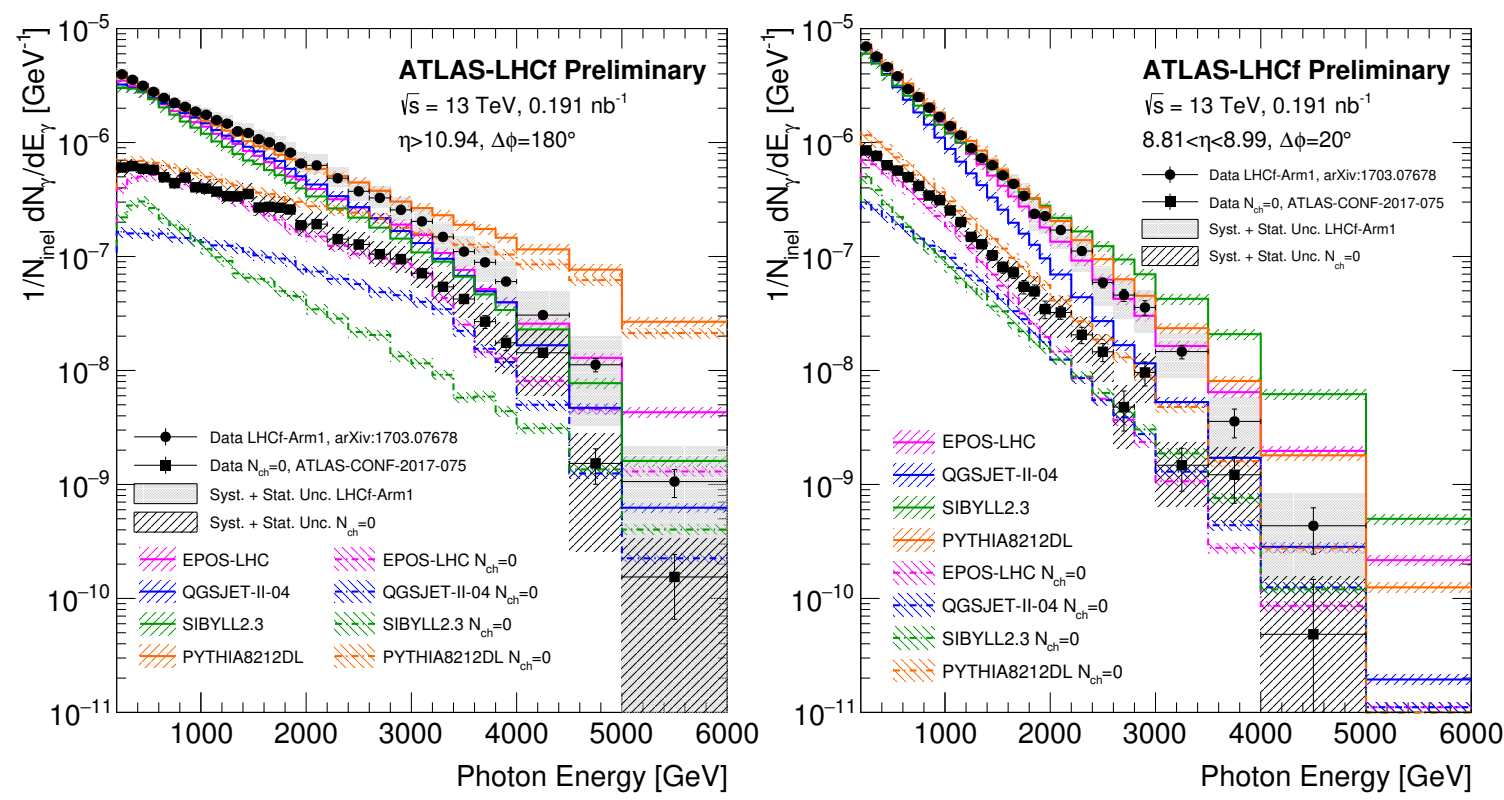

Figure 1: Preliminary photon energy spectra relative to $\sqrt{s}=13 \mathrm{TeV}$ p-p collisions, measured using the Arm1 detector and compared with model predictions [8]. Filled squares/circles show the experimental results with/without central veto $\left(\mathrm{N}_{\mathrm{ch}}=0\right)$, respectively. Vertical bars represent statistical uncertainties of the data sample, while gray bands indicate the quadratic sum of statistical and systematic uncertainties. Dashed/solid lines show the model predictions with/without central veto $\left(\mathrm{N}_{\mathrm{ch}}=0\right)$, respectively.

According to Monte Carlo studies [7], diffractive events can be identified with a purity of almost $100 \%$ and an efficiency of about $50 \%$ if we require a central veto, defined as zero charged tracks with $\mathrm{p}_{\mathrm{T}}>100 \mathrm{MeV}$ in $|\eta|<2.5$. Fig.1 shows the preliminary result relative to the Arm1 detector in the two pseudorapidity regions $8.81<\eta<8.99$ and $\eta>10.94$ [8]. Generators distributions are compared to experimental measurements, both with and without the central veto. Here we can note two important points. The first one is that, in both cases no model is able to reproduce satisfactorily experimental data in all the energy range. The second one is that the ability of a model to reproduce experimental data is different with or without central veto. For ex-

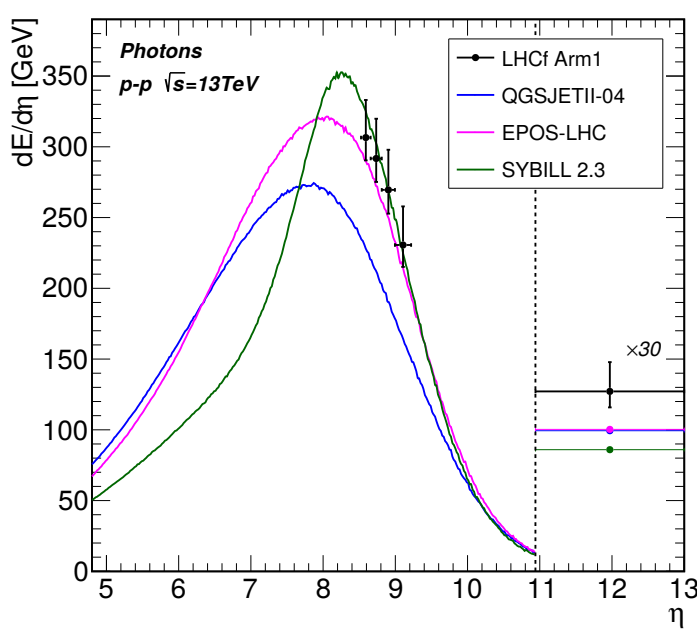

Figure 2: Preliminary photon energy flow relative to $\sqrt{s}=13 \mathrm{TeV}$ p-p collisions [10]. Black markers are the values measured using the Arm1 detector together with the quadratic sum of statistical and systematic uncertainties. Colored lines are model predictions from QGSJET II-04, EPOS-LHC, SIBYLL 2.3. ample, in $\eta>10.94$, QGSJET II-04 [9] is slightly softer than data without central veto, but with central veto is strongly softer at low energy. 
This with/without central veto discrepancy is expected for all models because diffractive and non diffractive mechanisms are treated separately inside them. It is therefore crucial to separately measure the role of the two contributions in forward particles production and this is the reason of the large activity dedicated to this analysis, that, after its extension to the Arm 2 detector, will be published soon. Another interesting result is the measurement of the photon energy flow as a function of pseudorapidity, that can be obtained using numerical integration on the inclusive spectra shown in Fig.1. In addition, thanks to the large acceptance of the Arm1 detector in the region $\eta<9.22$, it was possible to extend the analysis adding three points at small pseudorapidities. This is a very important point because it allows us to measure the energy flow near the region where it is expected to be maximum, that is, according to most models, around $\eta \sim 8$. Fig. 2 shows the preliminary photon energy flow [10], where we can see that, for $\eta>10.94$, no model agrees with data, whereas, for $8.52<\eta<9.22$, SIBYLL 2.3 [11] is in good agreement with the measurements, EPOS-LHC [12] is slightly softer but still compatible, while QGSJET II-04 leads to a significant underestimation.

\section{Conclusions and future prospects}

Thanks to the data taking relative to $\mathrm{p}-\mathrm{p}$ collisions at $\sqrt{s}=13 \mathrm{TeV}$, the LHCf experiment has now reached its main scientific aim. The analysis of this data set carried on so far showed that in the forward region no model agrees well with experimental observations. In addition, the LHCf-ATLAS joint analysis showed that it is possible to separate the contribution of diffractive and non-diffractive processes responsible for particles production in the forward region. Thus, LHCf measurements are expected to provide very precise information for the calibration of hadronic interaction models used by cosmic rays experiment. Regarding data relative to p-p collisions at $\sqrt{s}=13 \mathrm{TeV}$, photon results was already published, neutron analysis was just submitted for publication and $\pi^{0}$ activity was recently started. The LHCf experiment is also very interested in future p-O and O-O collisions, if, as it seems, they will be realized at the LHC during Run III.

\section{References}

[1] O. Adriani et al., Journal of Instrumentation 3 (2008) S08006.

[2] L. Evans, New Journal of Physics 9 (2007) 335.

[3] G. Aad et al., Journal of Instrumentation 3 (2008) S08003.

[4] T. Mase et al., Nuclear Instruments and Methods in Physics Section A A671 (2012) 29-136.

[5] K. Kawade et al., Journal of Instrumentation 9 (2014) P03016.

[6] O. Adriani et al., CERN Preprint (2017) CERN-EP-2017-051.

[7] Q. D. Zhou et al., Eur. Phys. J. C 77 (2017) 212.

[8] The ATLAS and LHCf collaborations, CERN Preprint (2017) ATLAS-CONF-2017-075.

[9] S. Ostapchenko, Physical Review D 83 (2011) 014018.

[10] Y. Makino et al., CERN Thesis (2017) CERN-THESIS-2017-049.

[11] F. Riehn et al., PoS ICRC 2015 (2016) 558.

[12] T. Pierog et al., Physical Review C 92 (2015) 034906. 